\title{
Le Réglage automatique hydraulique ultra-rapide des Fours électriques tourmentés
}

\section{INTRODUCTION}

DE L'UTILITÉ D'UN RÉGLAGE ULTRA-RAPIDE DES ÉLECTRODEs.

C'est un fait bien connu que la marche d'un four électrique à arc est caractérisée par des modifications continuelles dans l'état du bain et de son ambiant, provoquant des variations de la puissance absorbée par le four, qui sont plus ou moins importantes selon les produits traités.

Ces variations sont cause de multiples inconvénients : diminution $d u$ rendement $d u$ four, mauvaise utilisation des lignes des transformateurs et des générateurs, pointes sur le réseau, disjonctions, etc. Les fluctuations du bain, qui ne sont pas moins gênantes, se traduisent, à leur tour. par des variations de puissance, une usure excessive de l'électrode et, surtout, par une carburation du bain allant en général à fin contraire du but poursuivi.

Un ajustage de la position de l'électrode a donc une importance capitale qui incitera le chef d'usine à ne pas confier cette manœuvre à une main-d'œuvre coûteuse et incontrôlable, mais a avoir recours au réglage automatique. Le chimiste, d'autre part, se rendant compte de l'intérêt primordial qu'il y a, au point de vue de la qualité de la production, à s'assurer que les électrodes suivent immédiatement les fluctuations du bain et de l'intensité absorbée, ne se contentera pas d'un réglage automatique quelconque, mais donnera à un réglage ultra-rapide toute l'importance qu'il mérite.

DES CONDITIONS ESSENTIELLES D'UN RÉGLAGE ULTRA-RAPIDE.

La nécessité d'un réglage instantané de l'électrode conduit à rechercher l'élimination de toutes les causes de retards dans le réglage, retards qui sont essentiellement provoqués par l'inertie des masses à manœuvrer, l'inertie magnétique des organes électriques de commande et l'élasticité des organes de transmission.

Il est évident qu'il ne peut être question de chercher une atténuation de l'inertie des masses par une diminution du poids de l'électrode et de son armature ; c'est donc en premier lieu les organes de commande de l'électrode qu'il s'agit de réaliser d'une manière rationnelle. On proscrira donc, pour les fours qui exigent une grande rapidité de réglage, l'emploi des treuils commandés par moteurs électriques, à cause des roues, engrenages et rotors qui offrent une inertie énorme lors d'inversion du sens de réglage ; on rejettera aussi tout système basé sur l'excitation de machines à courant continu, par suite du retard à l'aimantation de ces machines. Enfin, on réduira au minimum la valeur du contrepoids, toujours dans le but de diminuer la masse du système.

Un seul mode de commande des électrodes répond entièrement à ces conditions essentielles de rapidité : celui par vérin hydraulique. Ce dernier est, en effet, un organe d'une souplesse parfaite, capable du plus grand travail en n'ayant qu'une inertie négligeable ; il permet la commande de l'électrode, soit directement, soit par câble, sans l'interposition d'organes intermédiaires.

D'autre part, il est évident que la commande hydraulique doit répondre aux exigences d'un réglage ultra-rapide par des dimensions convenables des tuyauteries, de manière à réduire au minimum la perte de charge lorsque le régulateur doit faire un brusque appel à toute l'énergie disponible.

Comme le mécanisme adopté pour la commande des électrodes, le régulateur automatique devra répondre lui aussi, aux exigences par sa propre rapidité d'action. Elle ne devra pas être entravée par l'inertie d'un organe de mesure de grandes dimensions, ni par l'effort que nécessite la commande du distributeur d'eau, alors même que toutes les conditions imposées par le réglage ultra-rapide concourent à l'adoption d'un distributeur d'eau de grandes dimensions nécessitant par conséquent un certain effort pour sa manœuvre. Cet effort, une construction logique ne l'imposera pas à l'appareil de mesure du régulateur mais à un servo-moteur auquel l'utilisation d'huile sous pression assurera une douceur et une sécuité de fonctionnement qui ne peuvent être obtenues, d'une manière très prolongée, que par l'utilisation de l'huile.

Le régulateur automatique Guénod type $\mathrm{HF}$ (fig. 1) réalise pleinement ces conditions, ainsi que l'on peut s'en rendre compte par la description suivante.

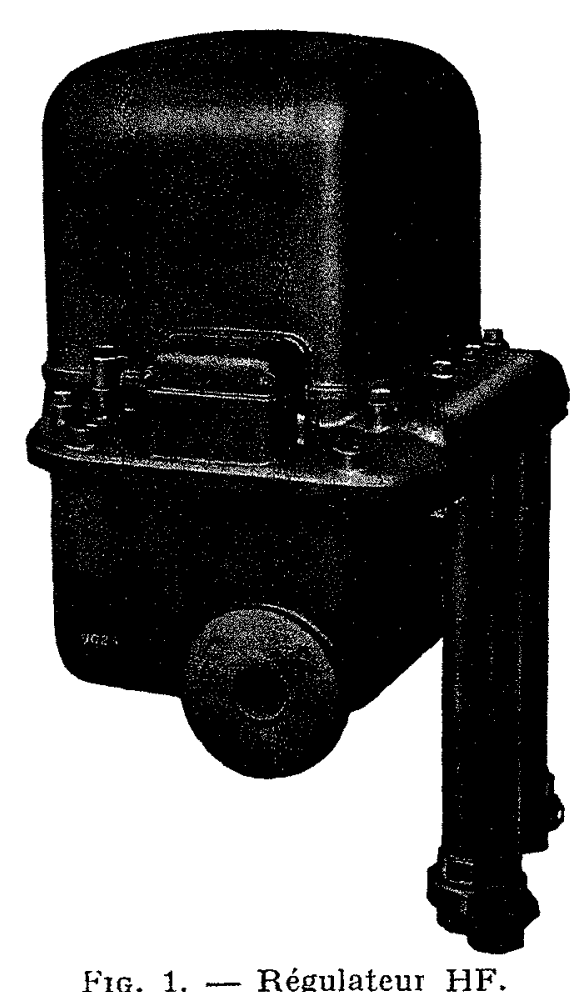

Fig. 1. - Régulateur HF. (côté boîte de conrexions)

\section{LE REGULATEUR HYDRAULIQUE GUENOU, TYPE HF}

Description : Le régulateur, dont on voit, à la fig. 2 une coupe schématique, comprend un solénoïde (6) mesurant l'intensité absorbée par le four et agissant sur l'obturateur (14) de la chambre (13) d'un servo-moteur à huile (5) alimenté par la pompe (10). Le piston (12) de ce servo-moteur (5) actionne, par le moyen du levier (16), le distributeur d'eau sous pression (2) de la pompe (3) alimentant le vérin (1) qui commande l'électrode à régler 
L'appareil comporte encore un dispositif de sécurité constilué par un solénoüde (7) mesurant la tension entre sole et électrode et commandant le distributeur (18) envoyant l'huile de part et d'autre du piston (19) soumis à l'cffort du ressort (20) et solidaire du levier (23) formant butée du piston (12).

Les écrous (9) et (24) réalisent des butées réglables, facilement accessibles, pour le servo-moteur (5), le distributeur (2) et le piston (19).

Fonctionnement : La fig. 2 rend facilement compréhensible le fonctionnement de l'appareil.

A l'état d'équilibre, c'est-à-dire lorsque l'intensité réglée est normale, le solénoïde (6) donne une position précise aux leviers (15) et une fuite déterminée à la cataracte (14) d'où il résulte dans les chambres (11) et (13) des pressions telles que le piston (12) est lui-même en équilibre ; le piston (2) ferme à ce moment les lumières du distributeur d'eau : l'électrode est immobile.
SOLÉNOIDE : Le solénoïde absorbe, à 50 périodes, 3,5 ampères sous 40 volts soit 140 voltampères. A cette consommation vient éventuellement s'ajouter celle de la résistance additionnelle dont l'importance est proportionnelle à la marge de réglage désirée.

Le solénoïde est formẻ d'un inducteur fixe à circuit magnétique feuilleté et d'un induit en court-circuit, rigide, indéformable, nullement sujet à avarie.

InSEnsibilité : Grâce à l'emploi du servo-moteur à huile, l'insensibilité du régulateur peut être extrêmement faible, moins de $1 \%$; une valeur aussi petite n'est, sauf certains cas particuliers, pas nécessaire en pratique ; elle peut du reste s'ajuster au banc d'essais du régulateur, à ce qui paraît le plus indiqué dans chaque cas.

Asservissement : Le régulateur possède un système amortisseur constitué par les leviers (15) et par un dashpot réglable. L'efficacité de ces dispositifs est telle qu'elle permet de garantir qu'aucun mouvement pendulaire de l'électrode n'est à craindre, même aux plus grandes vitesses de réglage.

\section{LE RÉGULATEUR TYPE HD}

Cet appareil ne diffère du modèle HF décrit ci-dessus que par le fait qu'il possède un distributeur d'eau (2) muni de 4 voies. Il permet donc l'envoi d'eau sous pression de part et d'autre du piston des vérins ou de la palette de servo-moteurs rotatifs.

On emploiera en particulier le régulateur $\mathrm{HD}$ pour la commande d'électrodes équilibrées ou horizontales (voir schéma fig. 9).

\section{DISPOSITIF DE SÉCURITÉ}

Chaque régulateur $\mathrm{HF}$ ou HD porte en lui-même les dispositifs de sécurité nécessaires, bloquant l'électrode en cas de manque de tension aux bornes du four ou de manque de pression d'huile. D'autre part, les vannes de réglage à main, dont il est question plus loin, comprennent un dispositif qui immobilise l'électrode en cas de manque de pression d'eau.

\section{Blocage Electromagnétioue.}

Lorsque, pourune raison quelconque, FIG. 2. - Représentation schématique du régulateur HF.
$\mathrm{Si}$, par contre, l'intensité absorbée par le four devient trop élevée, l'effort 'fourni par le solénoïde augmente et provoque une diminution de section de la cataracte (14), par conséquent une augmentation de pression de la chambre (13) par rapport à la chambre (11), donc un mouvement de bas en haut du distributeur (12), de haut en bas du piston (2) qui met en relation la pompe (3) et le vérin (1) : l'électrode monte.

Enfin, si l'intensité du four décroît, le ressort antagoniste du solénoïde provoque une augmentation de la fuite de la chambre (13), un abaissement de la pression dans cette dernière, un déplacement de haut en bas du piston (12), de bas en haut du piston (2) qui ferme l'orifice de la pompe (3), mais ouvre le vérin sur la décharge (4) : l'électrode descend par son propre poids. l'intensité ne peut plus être maintenue à la valeur ajustée, l'électrode tend à descendre dans le bain pour chercher à augmenter l'ampérage. C'est alors qu'intervient le blocage électromagnétique, qui empêche toute descente de l'électrode, lorsque la tension entre cette dernière et la sole se trouve être inférieure à une valeur déterminée, et ceci de la façon suivante :

Le ressort antagoniste du solénoïde (7) (fig. 2) provoque un mouvement de bas en haut du piston (18). L'huile sous pression passant alors par le conduit (17) pénètre dans la partie supérieure du piston (19). Ce dernier descend et soulève la butée (23) empêchant le piston (12) du servo-moteur (5) de descendre en dessous d'une cote déterminée. A cette cote correspond une position du piston (2) du distributeur d'eau telle que la conduite du vérin 
ne peut être ouverte sur l'évacuation. L'électrode ne peut donc descendre, bien qu'elle y soit sollicitée par le solénoïde principal ; par contre elle peut toujours monter. Lorsque la tension entre électrode et sole est redevenue normale, le solénoïde (7) fait descendre le piston (18); l'huile pénètre dans la chambre (21) et contrecarre l'effet du ressort (2) sur le piston (19). Le levier (23) se place dans la position indiquée sur la fig. 2 et laisse toute liberté au piston (12) d'effectuer la course complète nécessaire au réglage automatique.

\section{Blocage a Pression d'huile.}

En cas d'accident à la distribution d'huile ou d'arrêt du moteur la pression d'huile venantà manquer dans la chambre (21), le piston (19), poussé par le ressort (20), descend et provoque le jeu de la butée (23) qui empêche toute descente du piston (12) du servo-moteur (5) en dessous de la cote dont il est question dans le paragraphe précédent.

\section{Blocage hydRaulioue.}

Enfin, si par accident la pression d'eau vient à manquer l'électrode n'étant plus soutenue tendra à descendre dans le bain alors que le régulateur, cherchant à la faire remonter, mettra en communication le vérin (1) avec la pompe (3). L'eau contenue dans le vérin cherchera donc à s'écouler à travers la vanne de commande à main et par la pompe, dans le réservoir (voyez fig.2 ). Cette évacuation, et la descente de l'électrode qui en résulte, sont rendues impossibles par une soupape de retenue spéciale, montée dans la vanne à main et qui obture automatiquement la conduite venant du vérin, lorsque la pression d'eau tombe en dessous d'une valeur déterminée.

\section{ACCESSOIRES}

\section{Résistance Additionnelle.}

Il est indispensable de pouvoir modifier le régime du four pendant le cours d'une opération, cela, bien entendu sans interrompre le réglage automatique. Lors du réglage de l'intensité, on peut obtenir ce résultat, soit au moyen d'un transformateur, d'intensité sectionné, soit au moyen d'une résistance additionnelle insérée dans le circuit d'alimentation du solénoïde.

Cette dernière disposition permet l'obtention de régimes de réglage aussi nombreux et entre des limites aussi étendues qu'il peut être désiré.

Les résistances additionnelles normales possèdent 15positions réparties entre des limites comprises dans un rapport de 1 à 2,5; un rapport plus grand s'obtient au moyen d'une résistance suppiémentaire.

\section{Transformateur D'INTENSITÉ.}

Le transformateur d'intensité peut être monté sur la haute ou sur la basse tension du réseau alimentant le four. Lorsque la disposition des barres s'y prête et pour des intensités de l'ordre de 1.500 à 25.000 ampères, on prévoit un transformateur ne comportant pas d'enroulement primaire, celui-ci étant constitué par les barres elles-mêmes.

Pour des raisons de commodité d'installation, il pourra être indiqué, dans certains cas, de monter le transformateur sur la haute tension. Le choix se portera alors sur un appareil à air libre lorsque la tension du réseau est inférieure à 6.000 volts, à bain d'huile pour des tensions supérieures.

\section{Coffret de Manceuvre a main.}

Une attention toute spéciale a été donnée à la conception et à la réalisation de cet organe important dont le fonctionnement doit être très sûr (fig. 3).
Liaisons hydrauliques. - Le coffret possède 4 voies de forte section permettant d'obtenir, par la seule manœuvre du volant toutes les liaisons hydrauliques entre pompe, régulateur et vérin et de réaliser : $1^{0}$ l'immobilisation de l'électrode, $2^{0} \mathrm{sa}$ commande automatique par le régulateur $\mathrm{HF}, 3^{\circ}$ la montée et la descente indépendamment du régulateur.

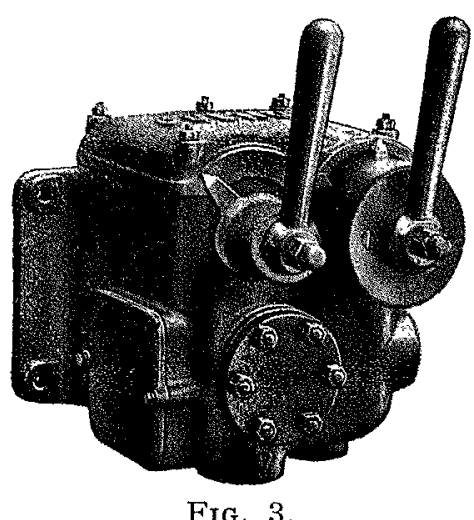

Vanne de manœurre à main.

L'immobilisation de l'électrode n'est pas réalisée par le boisseau du coffret, mais par une soupape fermant hermétiquement la conduite du vérin. Or cette soupape n'est pas placée seulement sous la dépendance de la commande à main, mais la fermeture est aussi provoquée automatiquement par un manque de pression dans la distribution d'eau (blocage hydraulique).

Cette disposition est particulièrement heureuse parce que le dispositif de sécurité, ainsi constitué, étant appelé à jouer à chaque manœuvre à main, on sera ainsi certain de son efficacité au moment opportun.

Connexions électriques. - Le coffret comporte encore les interrupteurs voulus pour court-circuiter le transformateur d'intensité et interrompre les circuits du blocage électromagnétique ou de tension du régulateur dans les positions autres que celles du réglage automatique.

Le régulateur $\mathrm{HF}$ est donc mis complètement hors service, électriquement et hydrauliquement, dans les positions de manœuvre à main et de repos du coffret.

\section{GRoupe MOTO-POMPES.}

L'installation de réglage hydraulique nécessite l'eau de sous pression pour le vérin et de l'huile sous pression pour le régulateur lui-même.

Alimentation d'eau. - L'ean peut être fournie par la distribution de l'usine ou par un réservoir dans lequel aspire une pompe centrifuge, elle doit être propre et ne pas déposer ; en général la seconde solution est choisie parce que plus pratique. La pression, si elle n'est pas déjà déterminée par une raison d'unification (raccordement à une chute existante, etc) dépendra du poids de l'électrode, éventuellement de son contrepoids et des caractéristiques de la suspension ; pour des électrodes de plusieurs tonnes on aura avanlage à adopter des pressions de l'ordre de 10 à $20 \mathrm{kgs} / \mathrm{cm}^{2}$ afin de diminuer la section des vérins ; par contre, pour des électrodes légères des pressions plus basses seront plus économiques. Le débit sera proportionnel au nombre d'électrodes, à la section des vérins et à la vitesse de réglage désirée.

Distribution d'huile. - L'huile ulilisée doit répondre à certaines conditions afin que l'on en obtienne toute satisfaction ; fluidité suffisante, viscosité aussi faible que possible (maximum $3^{\circ}$ Engler à $50^{\circ}$ C.), densité supérieure à 0,87 , pas d'acides, 
de résines ou d'asphaltes; il ne doit se former qu'un minimum de mousse. Il ne convient pas d'utiliser un mélange d'huile et de pétrole ; des essais prolongés permettent d'indiquer des marques d'huile donnant de bons résultats et se trouvant sur le marché mondial. La pression d'huile doit être de $1,5 \mathrm{~kg} / \mathrm{cm}^{2}$ et le débit de 1 litre-seconde par régulateur.

\section{GROUPE MOTO-POMPES.}

La solution la plus économique est constituée par un moteurentraînant d'une part la pompe à eau et d'autre part la pompe à huile. Cependant l'on peut aussi utiliser des groupes

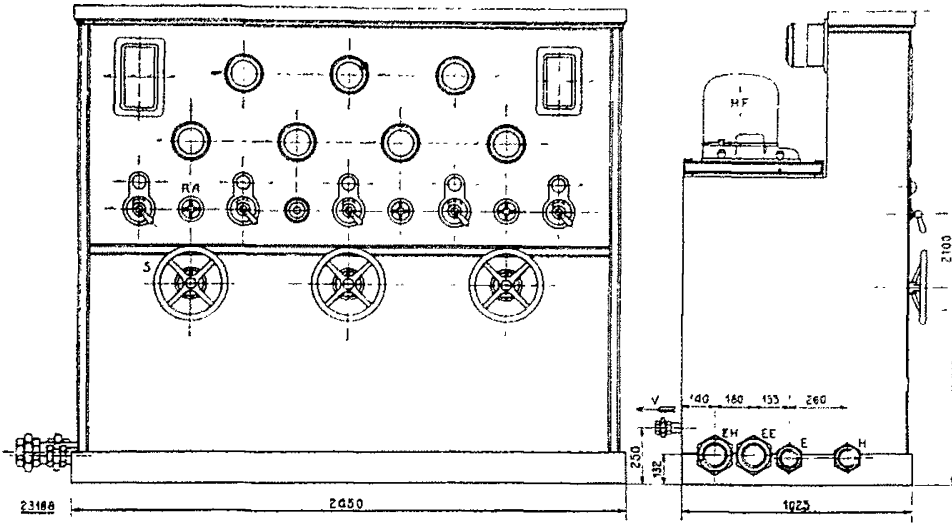

Fig. 6. - Tableau d'installation complet pour four triphasé. légende :

$R A$ Volant pour l'ajustage de l'intensité réglée. $H F$ Régulateur Guénod.

$E$ Tuyauterie d'amenée d'eau.

$E E$ Tuyauterie d'évacuation d'eau. etc.). Le régulateur type HD s'adapte à la commande de ces motcurs. séparés (fig. 4) et adopter, pour l'huile, un ensemble sans presse-étoupe grâce à un moteur vertical (fig. 5).

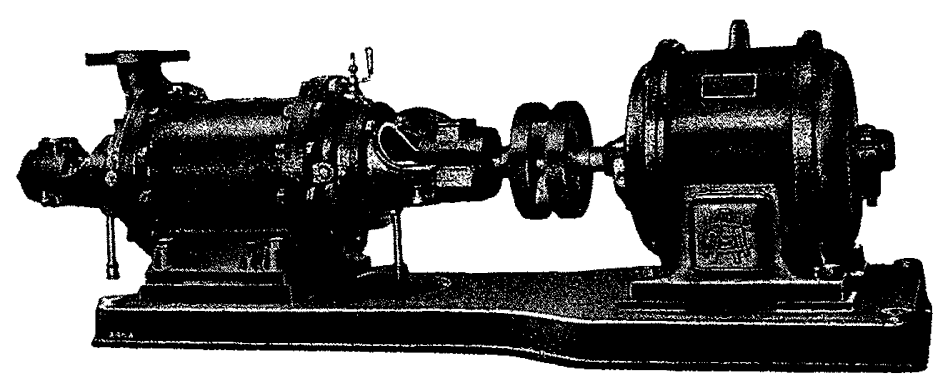

FIG. 4. - Groupe moto-pompe à eau.

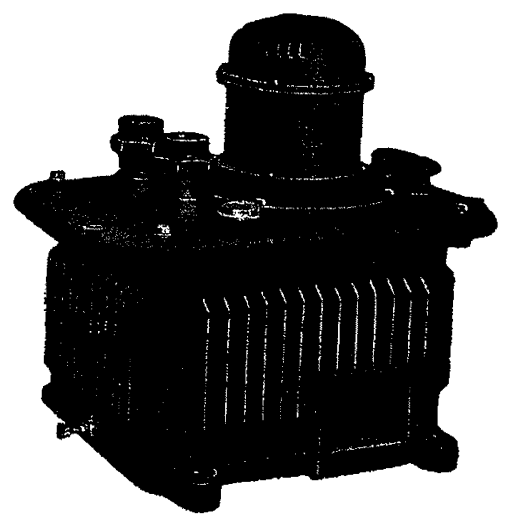

FIG. 5.

Groupe moto-pompe à huile.

\section{VÉRIN HYDRAULIQUE.}

Dans la généralité des cas l'électrode sera mue par un vérin hydraulique dont la section et la course dépendent des caractéristiques de l'installation. Cet organe, dont la simplicité est une garantie de robustesse, doit être construit et installé de façon à permettre sans difficultés le remplacement des garnitures usagées ; il possèdera les rejets nécessaires à Ja récolte des fuites (voyez fig. 7).

\section{MOTEUR HYDRAULIQUe.}

Ce genre de moteur peut avoir son utilité dans quelques cas (commande de treuils, d'électrodes équilibrées ou horizontales, $\mid \begin{array}{ll}S & \text { Volant de la vanne de mancuvre’à main. } \\ V & \text { Conduites allant aux vérins des électrodes. } \\ H & \text { Tuyauterie d'an'enée d'huile. } \\ E H & \text { Tuyauterie d'évacuation d'huile. }\end{array}$

Poste de Commande.

Un pupitre ou un tableau de commande donne une solution pratique au groupement des organes de contrôle et de manœuvre d'un four.

La figure 6 représente un tableau destiné à J'installation d'un four triphasé. Les grands volants visibles sur ces figures sont ceux des vannes de manœuvre à main, les petits volants commandent les résistances d'ajustage de l'intensité réglée.

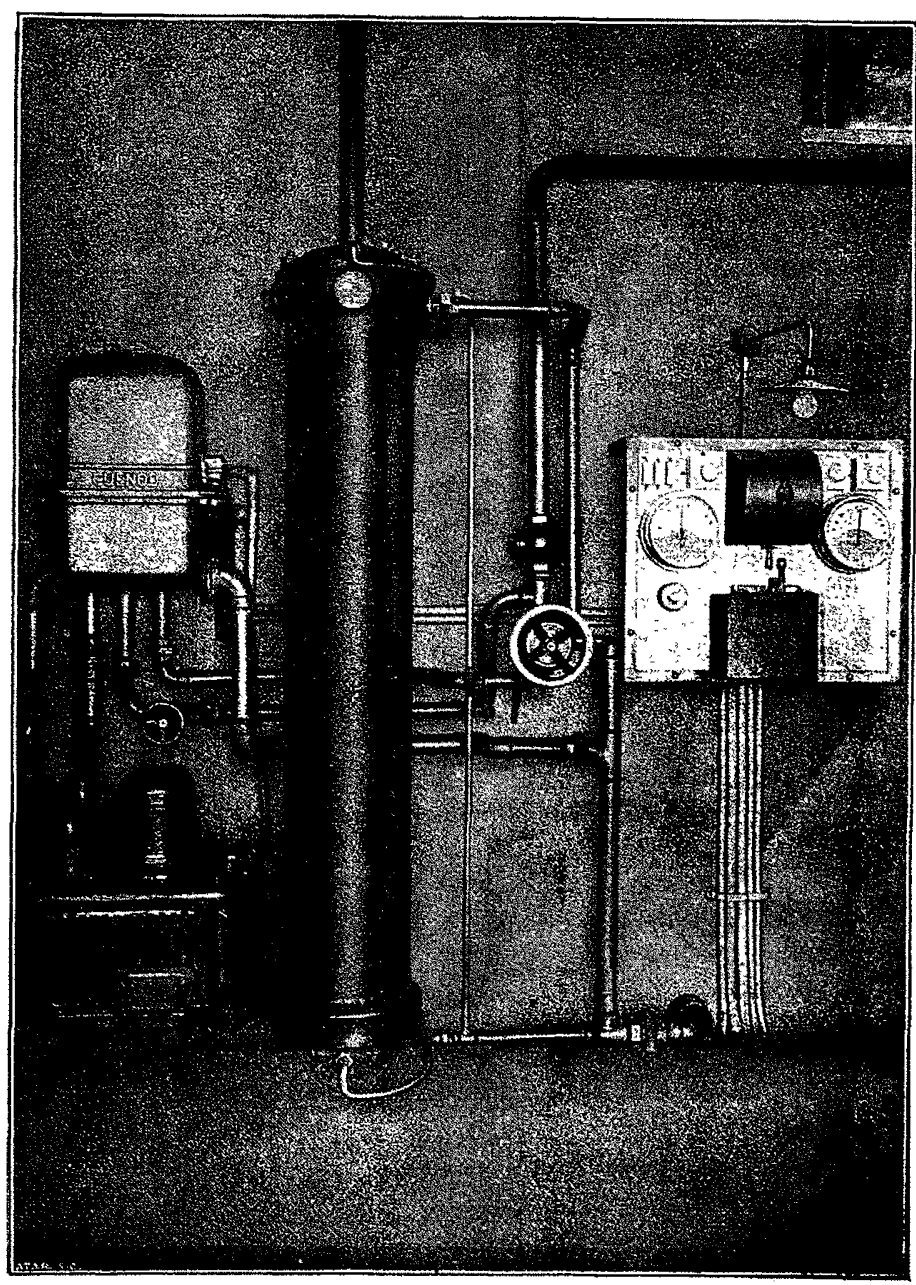

FIG. 7. - Vue d'une installation type. 


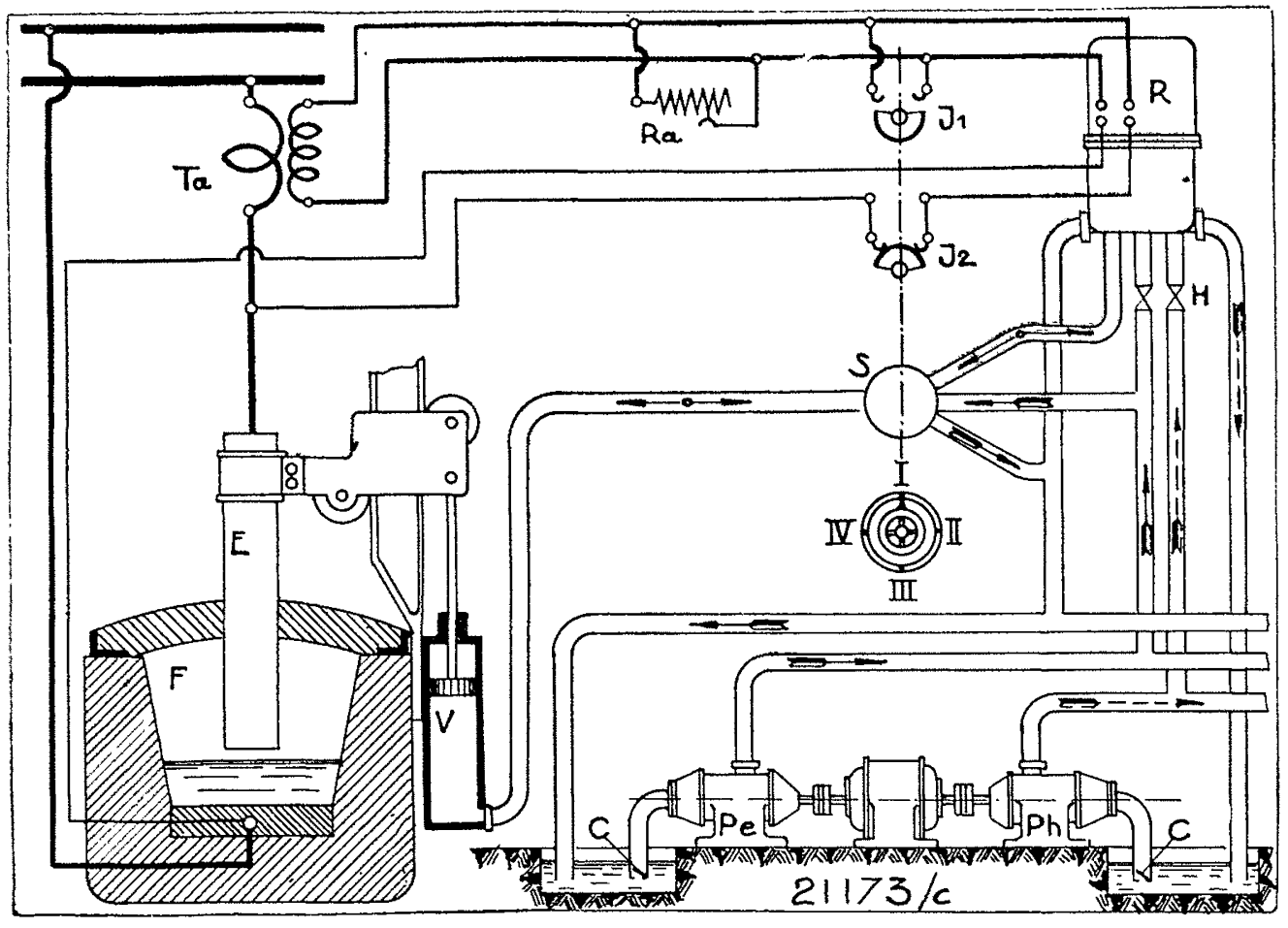

FIG. 8. - Schéma de réglage de l'intensité d'un four électrique (schéma pour une électrode).

$F$ Four.

Légende :

$V$ Vérin.

IR Régulateur.

$S$ Vanne de commande à main cl blocage hydraul. I. Position réglage autom.

II. Position montée.

III. Position arrêt.

IV. Position descente.

E Electrodes.

Ta Transformateur intensité.

Ra Résistance ajustage intensité.

$I_{1}$ Interrupteur de court-circuit.

$I_{2}$ Interrupteur du blocage.

$H$ Vannes.

Pe Pompe à eau.

$P h$ Pompe à huile.

$C$ Clapet de pied.

\section{INSTALLATION D'UN ÉQUIPEMENT DE RÉGLAGE}

Composition D'Un EQUipement.

Il comporte d'habitude pour chaque électrode: le régulateur $\mathrm{HF}$, la résistance additionnelle d'ajustage au régime voulu, la vanne de manœuvre à main, le vérin hydraulique, le groupe moto-pompes eau-huile, enfin éventuellement le transformateur d'intensité ou le shunt. A ceci s'ajoutent les organes de contrôle $\mathrm{du}$ four qui ne sont pas particuliers au réglage automatique.

\section{InstaLlation - Montage.}

La fig. 7 montre un ensemble de réglage groupé dans un espace limité ; à gauche, le régulateur HF se trouve au-dessus du groupe fournissant la pression d'huile; au milieu le vérin hydraulique avec sa tige commandant l'électrode par câble; à droite du vérin la vanne de mannuvre à main, plus à droite encore, le tableau des appareils, audessus duquel se trouve la conduite d'arrivée d'eau sous pression et, tout en bas, l'évacuation de l'eau.

Comme on le voit, le régulateur se fixe au moyen de consoles contre une paroi ou un cadre en fer ; étant complètement blindé il peut être placé dans la salle des fours. La vanne est fixé habituellement sur des fers cornières, mais peut aussi être disposée pour être fixée sur le sol au moyen d'un pied spécial livré sur demande.

Les connexions électriques et les liaisons hydrauliques sont à exécuter conformément aux schémas remis.

Une disposition pratique d'un équipement est donnée par le tableau de la fig. 6, tableau portant tous les instruments de mesure, de contrôle, de manœuvre a main et de réglage automatique d'un four triphasé. L'installation complète ne nécessite que l'établissement des connexions électriques aux transformateurs et disjoncteurs et les liaisons hydrauliques aux vérins et groupe moto-pompes.

F Four.

$V_{1} V_{2}$ Vérins.

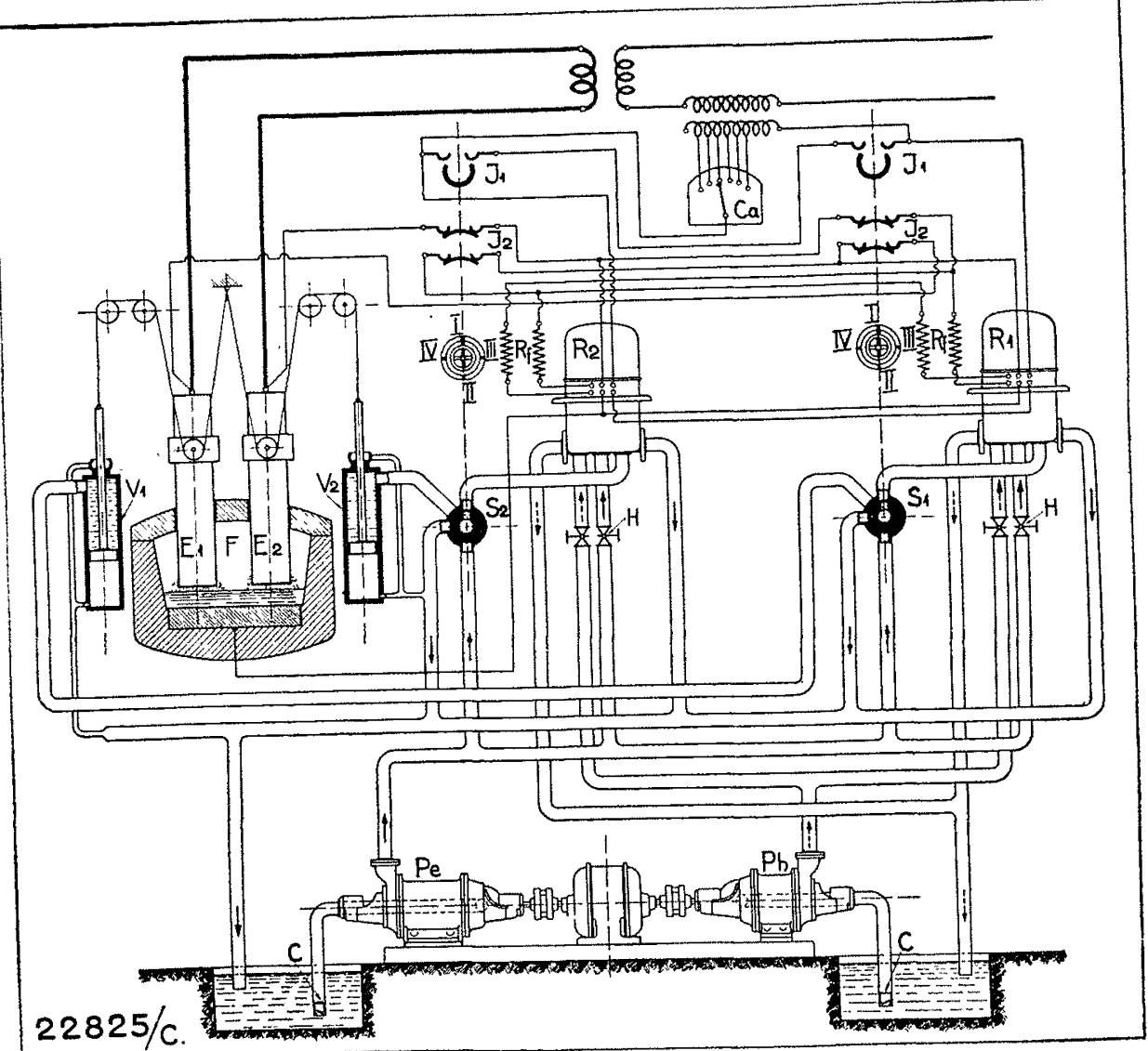

Frg. 9. - Schéma de réglage automatique de l'intensité et de la tension d'un four à 2 électrodes série.

Légende :

$E_{1} E_{2}$ Electrodes.

Ta Transformateur intensité.

$R_{1} R_{2}$ Régulateurs.

$\mathrm{Ca}$ Commutateur ajustage intensité

Rf Résistances fixes.

$S_{1} S_{2}$ Vannes de commande à main et blocage hydraul. Position réglage automatique.

\section{APPLICATION DU RÉGULATEUR HF}

DES DIVERS MODES DE RÉGLAGE.

Le régulateur $\mathrm{HF}$ peut être équipé pour n'importe quel mode de réglage: intensité, tension, puissance ou impédance - et pour toutes les combinaisons de ces régimes.

Le réglage à intensité constante est en général suffisant et, à le fournisseur d'énergie parce que le four joue alors le rôle de volant lors de variations de tension du réseau de distribution. 


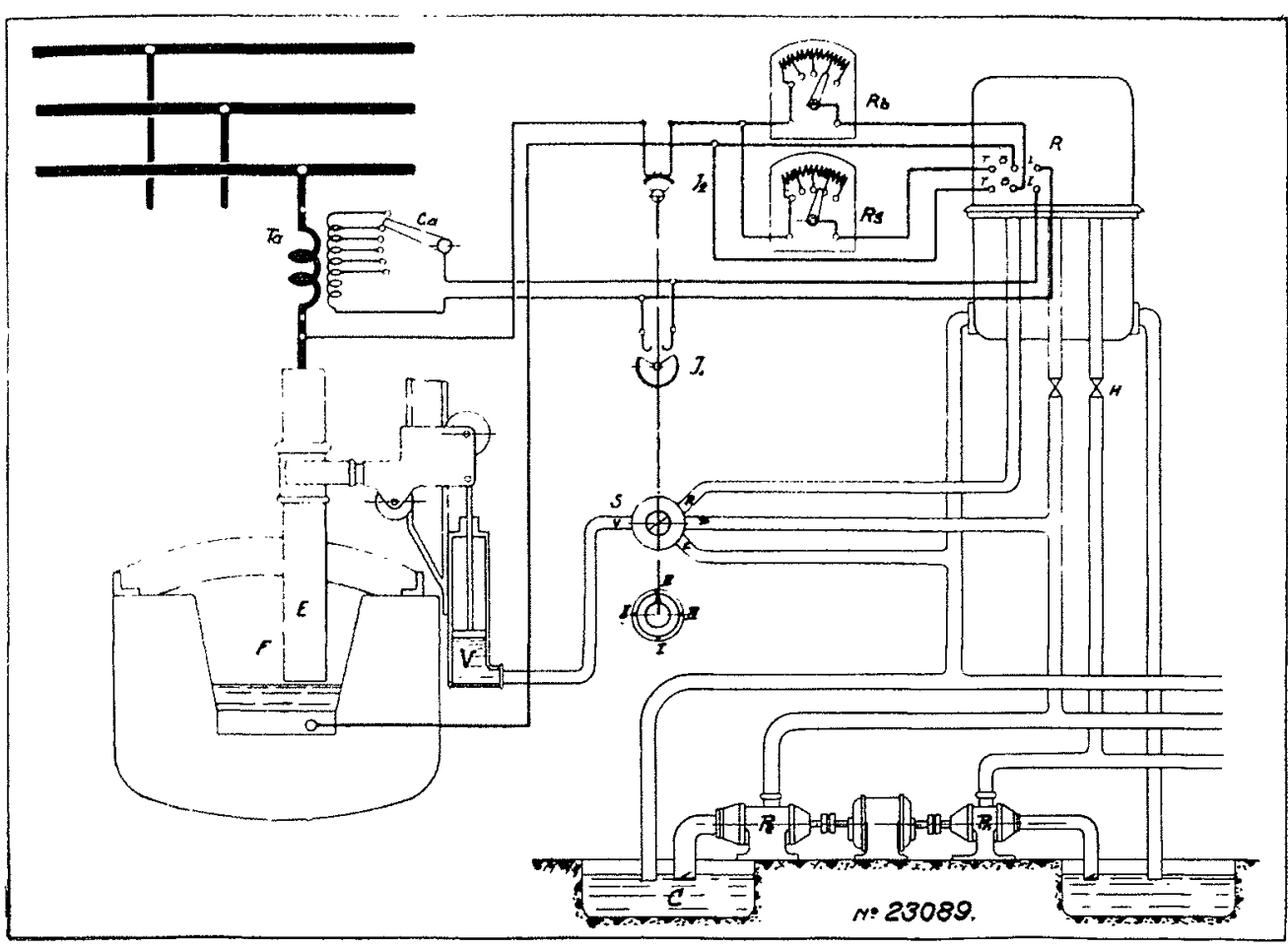

:Fig. 10. - Schéma de réglage automatique d'impédance d'un four triphasé (schéma pour une électrode). Légende :

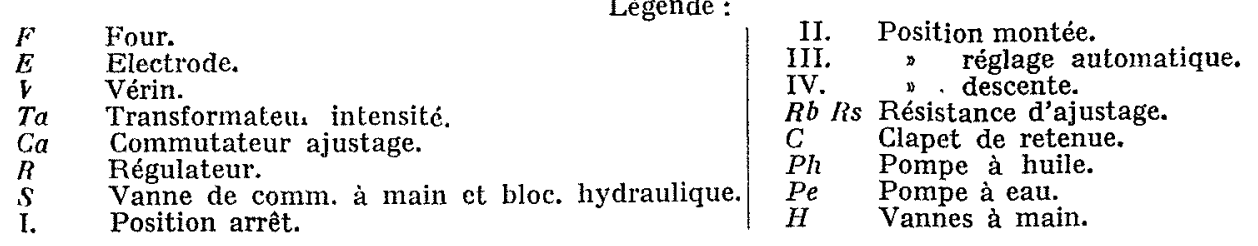

soumises à l'action du blocage électromagnétique.

\section{Four Biphasé.}

Chaque électrode sera soumise à un réglage d'intensité comme celle d'un four monophasé, par conséquent selon schéma fig. 8 .

\section{Four Triphasé.}

Réglage d'intensité. - Chaque électrode sera réglée individuellement, c'est-à-dire aussí selon schéma fig. 8. Si la sole est accessible le blocage électromagnétique sera branché entre celle-ci et chaque phase.

Réglage d'impédance. - La fig. 10 montre le schéma de réglage d'impédance de l'arc de chaque électrode qui peut être réalisé sans aucune difficulté quelconque par les régulateurs $\mathrm{HF}$.

Four a Électrodes horizontales.

Ce genre de four sera réglé au moyen du régulateur type HD à distributeur d'eau à 4 voies. La fig. 11 donne le schéma de connexions pour une électrode.

\section{CONCLUSIONS}

Avantages DU R̨Églage Ultra-rapide.

Le bon rendement du four, la qualité des produits traités, la sécurité de fonctionnement des installations dépendent essentiellement d'une très grande rapidité de réglage.
Le réglage à puissance constante est indiqué si le réseau est sujet à de fortes variations de tension et que le métallurgiste lienne cependant à un débit régulier de son four.
Le bon rendement résulte du fait que les générateurs et transformateurs, ainsi que le four lui-même, peuvent être utilisés non seulement à leur pleine puissance mais surtout à puissance
Enfin le réglage d'impédance, pour les fours triphasés, est précieux dans certaines fabrications. On remarquera cependant qu'avec ce mode de réglage les variations de la puissance absorbée sont proportionnelles au carré des variations de tension éventuelle du réseau.

Les schémas de réglage automatique varient suivant les fours et le genre de courant ; voici les plus usuels :

Four monophasé A 1 Électrode.

Le schéma fig. 8 indique les appareils et connexions nécessaires au réglage à intensité constante.

Four monophasé a 2 Électrodes EN SÉRIE.

Le réglage d'intensité seule ne peut convenir pour ce genre de four ; il s'agit en effet de réaliser en plus un partage égal de la tension sous chaque électrode; le schéma fig. 9 donne une solution parfaite de ce problème. Chaque régulateur HF n'intervient pas seulement pour corriger les variations del'intensité mais encore de la tension sous chacune des 2 électrodes. Ces dernières restent

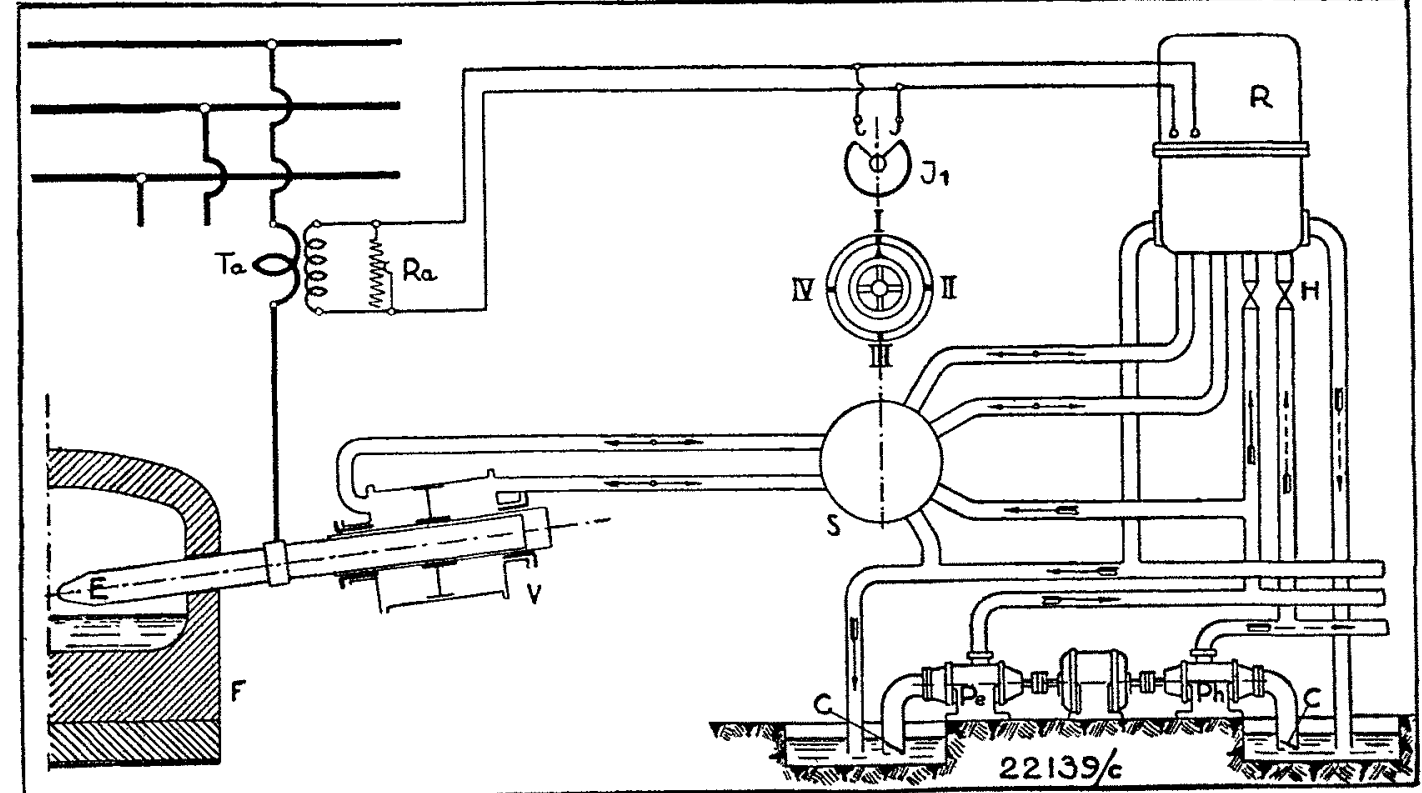

FIG. 11. - Schéma dèréglage automatique de l'intensité d'un four à électrodes horizontales (schéma pour une électrode).

\section{Légende :}

\begin{tabular}{ll|}
$F$ & Four. \\
$E$ & Electrode. \\
$C$ & Vérin. \\
$T a$ & Transformateur intensité. \\
$R$ & Régulateur type HD. \\
$R a$ & Résistance ajustage intensité. \\
$S$ & Vanne de commande a main et bloc. hydraul. \\
I. & Position réglage automatique.
\end{tabular}

$\begin{array}{ll}\text { II. } & \text { Position montée. } \\ \text { III. } & \\ \text { IV. } & \text { arrêt. } \\ I_{1} & \text { Intescente. } \\ H & \text { Vannes. } \\ P e & \text { Pompe à eau. } \\ P h & \text { Pompe à huile. } \\ C & \text { Clapet de pied. }\end{array}$


constante. Les constatations d'exploitation d'un four à régime tourmenté ont montré que le réglage ultra-rapide avait permis de réduire de $10 \%$ l'intensité absorbée sans diminuer la production du four.

La qualité des produits est assurée par la grande rapidité de réglage, qui empèche l'électrode d'entrer en contact aver lo bain ou, tout au moins, ne le permet que pendant le temps strictement nécessaire pour assurer l'amorçage do l'are. Il en résulte une régularité très grande du produit traité et une pureté remarquable du produit affinć qui n’est pas contaminé par l'électrode.

La sécurité de fonctionnement provient, dies l'amorçagé, de la correction immédiate de toutes les pointes de courant, donc protection des installations contre les surcharges accidentelles, abolition des déclenchements si désastreux des disjoncteurs, suppression enfin d'une main-d'ouvre plus ou moins consciencieuse et difficilement contrôlable.

Ces avantages ne sont assurés que par un réglage ullra-rapide des électrodes, c'est-à-dire de l'ordre de grandeur de $150 \mathrm{~mm}$. par seconde.

\section{Avantages du RÉgulateur Guénod HF.}

Le régulateur ultra-rapide Guénod HF n'est pas un appareil " universel " adapté, avec plus ou moins de succès, au réglage hydraulique des fours électriques. Tous ses organes ont été étudiés et exécutés spécialement en vue d'un réglage ultra-rapide des électrodes. Ce réglage demande tout particulièrement une action immédiate, un effort moteur élevé, et, comme cela a été déjà exposé, une robustesse à toute épreuve de l'appareil de réglage dont la sensibilité doit cependant rester très fine. - Ces 4 caracteristiques ne sont généralement pas conciliables; en effet, la sensibilité et la rapidité d'action ne peuvent guère être attendues que d'un appareil de réglage dans leque! l'effort à fournir est aussi limité que possible. I a sensibilité n'est du reste pas généralement le fait d'un appareil robuste, dont les masses ont une inertic défavorable à unc grande vitesse de réglage. Ce n'est que par l'cmploi d'un scrvo-moteur à huile qu'il a été possible de réaliser simultanément cés 4 conditions qui, autrement, réagissent défunorablement lune sur l'autre. Crrâce à co servo-moteur, le régulateur (iuénod $1 \mathrm{ll}$ : possède un solénoüde de dimensions réduites ayant une inertie négligeable; toute la marge de réglage s'effectue sur une course de $5 \mathrm{~mm}$.; l'effort à fournir par ce solénoïde est minime et absolument indépendant de l'effort résistant du distributcur d'eau sous pression.

1)'autre part, leffort puissant, pouvanl atteindre $30 \mathrm{~kg}$., à disposition pour la commande du distributeur d'eau a permis d'exécuter cet organe sur des bases excessivement larges; les sections de passage de l'eau sont grandes et l'appareil se prête à l'utilisation de pression d'eau jusqu'à 25 atmosphères. De plus, la course du distributeur n'étant que de $2 \mathrm{~cm}$., les effets d'inertie de cet organe sont nuls.

Ce distributeur d'eau est monté dans le carter mème du régulateur, et la transmission des mouvements, entre les différents organes, s'opère par des lames flexibles et non au moyen de leviers munis d'articulations qui sont sujets à une prompte usure.

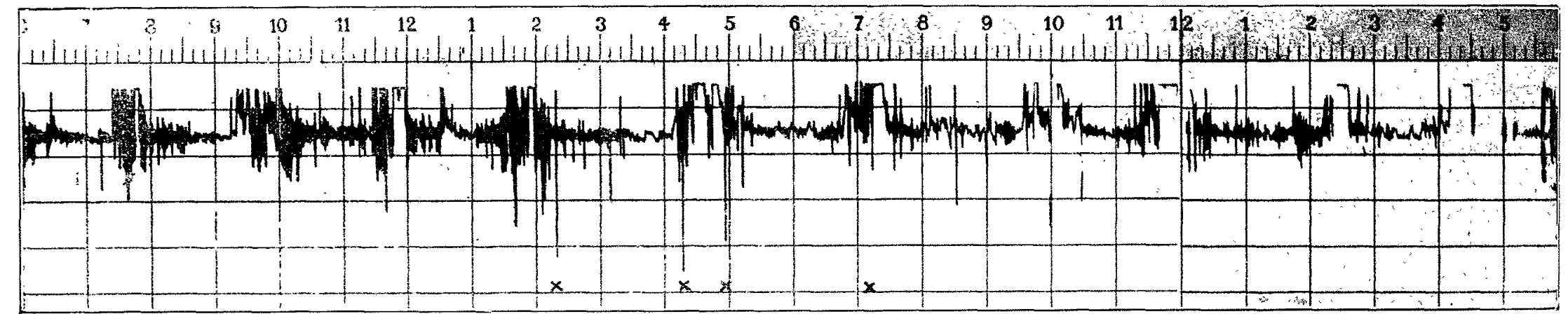

Fig. 12. - Diagramme de lintensité d’un four réglé automatiquenent par moleur électrique et treuil. - Vitesse de réglage 60 cm/min. $\times$ Disjonction.

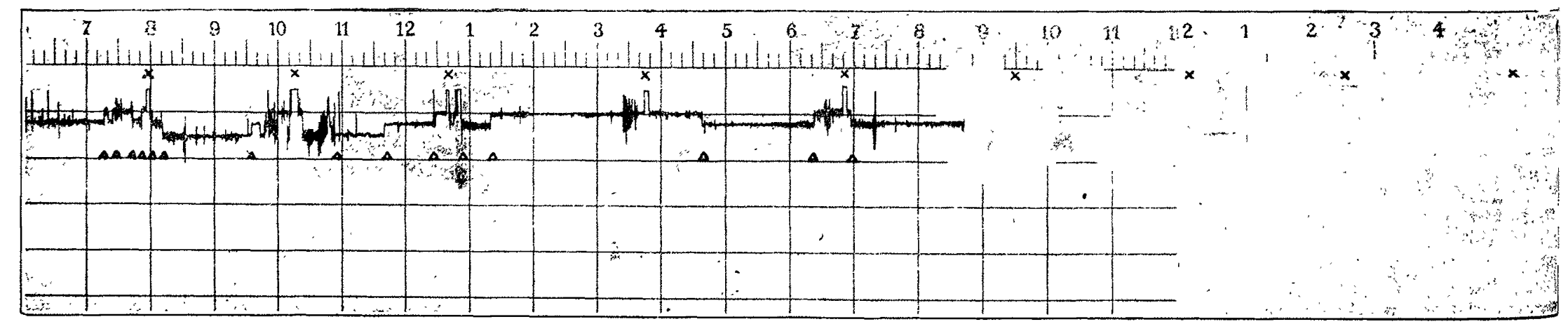

Fı́. 13. - Diagramme de l'intensité du mème tour, mais muni d'un régulateur ultra-rapide Guérod HF. - Vitesse de réglage $8 \mathrm{~m} / \mathrm{min}$, $\Delta$ Modification volontaire à l'intensité ajustée.

$X$ Arrêt du four. 
En résumé, le régulateur Guénod type HF conslitue le régulateur de four le plus rapide, le plus précis et le plus robuste actuellement connu; le plus rapide par la suppression de tous effets d'inertie pouvant retarder l'action du solénoïde et des distributeurs et par les dimensions très larges de ces derniers organes, permettant la commande des électrodes les plus lnurdes aux vitesses les plus rapides; le plus précis grâce an fait que le solénoïde, mesurant la grandeur réglée, n'a pas d'offorl appréciable à vaincre puisqu'jl commande le distributeur par servomoteur ; le plus robuste, par sa conception moderne, par ses organes commandés par lames flexibles et par sa construction entièrement blindée.

\section{Diagramates de Ríglagit.}

Les diagrammes fig. 12 et 13 ont élé relevés sur le mème four et pour le même produit traité ; le régime de ce four peut être considéré comme le plus tourmenté qui se rencontre en métallurgie.
La firs. 12 indigue les varialions de l'intensite, lo róglage antonatique du four agissunt sur l'́lectrode par moteur électrique et treuil à raison de $60 \mathrm{~cm} /$ minute. On constate que la vitesse de réglage ne permet pas d'éviter des à-coups qui font intervenir le disjoncteur automatique.

La fig. 13, par contre, relève les variations de l'intensité absorbée par ce mème four alors qu'il est régli automaliquement par régulateur ultra-rapide Ciuénod IIF, agissant sur un périn permellant un déplacement d'électrode de $8 \mathrm{~m}$. par minule.

L.a comparaison de ces deux diagrammes illustre d'une facon farticulièrement frappante les avantages du réglage hydraulique Lrès rapide réalisé par le régulateur Guénod HF.

Les particularités du régulal cur " Cuénod HF"permet d'assurer un réglage ultra-rapide des fours à régime tourmenté, on même simplement d'obtenir une grande régularite de marche. Il est encore avantageux pour le réglage, à vitesse réduite, d'électrode de 10 à 50 tonnes, parce qu'il conduit à une grande simplification de leur commande mécanique.

\section{DOCUMENTATION}

\section{Installations électriques de la "Direttissima, Firenze - Bologna"}

L'importance de celte nouvelle artère est grande, tant au point de vue du décongestionnement du trafic entre deux régions italiennes économiquement les plus importantes, qu'au point de vue technique. Car, en effel, l'Italie est une des nations curopécinnca où l'électrification des chemins de fer est le plus activement.pousséc. Tous les systèmes d'électrification y ont été essayés. L'option, qui semble définitive, pour le courant continu $3.000 \mathrm{~N}$, est le résultat de longues études et d'observations, facilitées par les résul. lats obtenus sur des réseaux différemment équipés.

Ayant déjà eu l'occasion de fournir, ici même, une pelite étude sur les difficultés surmontées pour mener à bien cette réalisation intéressante du Génie Civil (1), nюus nous proposons de donner, aussi brièvement que possible, quelques renseignements sur les installations électriques qu'elle comporte.

Alimentation en énergie électrique. - L'ancienne ligne électrifiée en triphasé de la Porrettana qui reste toujours en service disposait d'une source d'énergie d'environ $35 \times 10^{6} \mathrm{kWh}$, pour faire face aux nouvelles nécessités de force motrice, les équipements $d_{0}$ Suviana et de l'Alto Reno mettent à leur disposition une nouvelle source d'énergie de $45 \times 10^{6} \mathrm{kWh}$. Les $80 \times 10^{6} \mathrm{kWh}$ par an dont on dispose sembilent largement suffisants pour les besoins du trafic régulier en Firenze et Bologna. En cas d'une demande supérieure en énergie, un réseau d’interconnexion permet facilement l'apport de nouvelle force motrice provenant des réseaux ligure, toscan et émilian.

Les lignes d'amenée de haute tension ont été l'objet d'études particulic̀rement soignées afin d'être aussi sûr que possible de la régularité de leur fonctionnement, même pendant les tourmentes: les plus graves dont la région est fort souvent gratifice.

La tension de transport généralement adoptée est $60 \mathrm{kV}$, sauf pour la ligne principale, qui, à cette occasion, a vu sa premièro partie se réaliser, où elle est à $130 \mathrm{kV}$, el sera appeléo à l'avenir à joner le rôle " d'épine dorsale " du réseau général d'interconnexion des lignes à haute tension italienne.

Les pylônes emplloyés pour le soutènement de ces lignes à haute tension sont de types différents répondiant aux exigences de chaque région, oì ils devaient être installés :

(1) La Direttissima, Bologne - Florence, par G. Kolovitch. La Houille Blanche, Novembre-Décembre 1934. a) Pylones à treillis tronc-pyramidaux : ils comportenl cinq types dilférents dont les caractéristiques dépendent de la longueur Le la próe qu'ils sont destinés à former ainsi que de l'angle qua la ligne lait à leur sommet. Le taux de travail pour les treillis tranaillant à la compression a été déterminé par les formules des F.F.S.S. (chemins de fer italiens de l'Etat). Fn indiquant par $\sigma$ la charge maxim. à la compression simple el $\sigma_{p}$ relle à la compres. sion et flexion on a :

$$
\begin{gathered}
\text { pour } \frac{l}{r} \leqslant 30 \quad \sigma_{\mathrm{p}}=12 \mathrm{~kg} / \mathrm{m}^{2} \\
\text { pour } 30<\frac{l}{r}<105 \quad \sigma_{\mathrm{p}}=\left(12,07-0,0069 \frac{l}{r}\right) 12 \\
\text { pour } \frac{l}{r}<105 \quad \sigma_{\mathrm{p}}=\frac{5.300}{\left(\frac{l}{r}\right)^{2}} 12
\end{gathered}
$$

oì $l$ est la longueur libre du treillis et $r$ son rayon de giration, Le taux maximum des rivets et boulons ponr des charges normales a été fixé à $9 \mathrm{~kg} / \mathrm{mm}^{2}$ et à $12 \mathrm{~kg} / \mathrm{mm}^{2}$ pour des charges exceptionnelles.

Les fondations des pylònes sont en béton et ciment du type plcin ou creux, suivant le cas. Dans leur calcul on a tenu compte lì où cela était possible, de lla résistance Iatérale du terrain.

En partant des équations caténaires on a déterminé les filèches aux diférentes tempéralures et cfforts de tension, ce qui a permis diaborer des tableaux fort utiles pour la pose et tension des conducteurs.

b) Pylònes à treillis du type à chevalel : en conséquence de leurs dimensions et poids très élevés, ils ont été montés en troncons séparés el boulonnés sur place. Dans la mesure du possibje on a cherché à adapter la forme de la base à la configuration dı lerrain. Dans cette série, comme alins la précédente, il $v$ a des ly pes différents dont les caractéristiques varient suivant la longueur de la portée qu'ils vont constituer, ainsi que de J'angle qua la ligne forme a leur sommet. Les angles pour lesquels le type de pylône à employer change, sont $5^{\circ}, 16^{\circ}, 30^{\circ}$ et $60^{\circ}$.

Leur calcul a été fait en partanl des mêmes principes que pour les précédents, seule la surcharge due à la glace a été portée à $2 \mathrm{~kg}$. par mètre courant. 